\title{
Challenging Case in Clinical Practice: Challenging Conventional Wisdom in Type 2 Diabetes Mellitus- Does Every HgbA1c Above 9.0\% Require Insulin? An Integrative Approach to Type 2 Diabetes
}

\author{
Kendall Smith, ND, Jacob Hwang, ND, \\ Shawna McNally, MSPH, RD, and Ryan McNally, ND, MS, PA
}

\begin{abstract}
Type 2 diabetes mellitus (T2DM) is a disease characterized by endocrine dysfunction with metabolic derangements. These are primarily composed of peripheral insulin resistance and, eventually, inadequate insulin secretion. Excess adiposity often precedes this condition, in part as a result of consuming highly processed foods and living a sedentary lifestyle. Compelling scientific evidence indicates that lifestyle and dietary modification effectively treats, prevents, and delays the development of T2DM. Establishing individualized methods to prevent T2DM is an urgent challenge for our health care community and society. This case highlights the utilization of integrative modalities such as nutritional, lifestyle, and nutraceutical interventions and demonstrates a novel approach in the long-term maintenance and prevention of T2DM.
\end{abstract}

Keywords: type 2 diabetes mellitus, dietary modification, lifestyle intervention, insulin, metformin, berberine

\section{Background}

Type 2 diabetes mellitus (T2DM) is a common disease affecting $>382$ million people worldwide, a statistic that is in-

(C) Kendall Smith et al., 2019; Published by Mary Ann Liebert, Inc. This Open Access article is distributed under the terms of the Creative Commons License (http://creativecommons.org/licenses/ by/4.0), which permits unrestricted use, distribution, and reproduction in any medium, provided the original work is properly cited. creasing annually. ${ }^{1}$ It is defined as an endocrine disorder characterized by elevated blood glucose, HbA1c, and lipid levels, resulting from insulin resistance. T2DM increases the risk of cardiovascular, renal, and neurological complications and has become one of the most prevalent causes of morbidity and mortality in patients $<60$ years of age. ${ }^{1}$

Obesity, a result of energy imbalance, is the primary risk factor for T2DM. Coinciding with globalization and urbanization, household and workplace activities became more sedentary, leading to a reduction in energy expenditure. Likewise, an increased consumption of ultraprocessed foods and sugary beverages provides high caloric content and low nutritional yield, which result in excess energy consumption. The resulting energy imbalance coupled with genetic predisposition has created a global obesity epidemic and consequently, increasing rates of T2DM.

Recent literature demonstrates the importance of incorporating individualized dietary and lifestyle changes in the management of T2DM. ${ }^{2,3}$ A meta-analysis of 16 studies investigating the effects of diet, physical activity, and education interventions in the management of T2DM revealed a significant reduction in body mass index, $\mathrm{HgAlc}$, and blood pressure in the intervention groups as compared with the control groups. ${ }^{3}$ Likewise, a Cochrane review concluded that diet and physical activity reduced the incidence of T2DM in people with impaired glucose tolerance. ${ }^{2}$ Several studies determined that incorporating a Mediterranean diet improved glycemic control, weight management, and blood lipids in patients with diabetes. ${ }^{1}$

The literature suggests that supplementation with certain nutraceuticals may aid in improving metabolic disorders seen in T2DM. For example, berberine, an alkaloid derived from medicinal herbs such as goldenseal (Hydrastis canadensis), 
barberry (Berberis vulgaris), and Oregon grape root (Mahonia aquifolium), has been demonstrated to be safe and effective in lowering blood glucose, increasing insulin sensitivity, and reducing blood glucose, lipids, and triglyceride levels. ${ }^{4}$ Treatment with berberine $50 \mathrm{mg} / \mathrm{kg}$ per day for 2 weeks was found to be effective in vivo against the features of insulin resistance syndrome, including improved levels of insulin resistance parameters, lipid profiles, antioxidant enzymes, proinflammatory cytokines, and interferon- $\gamma .{ }^{5}$ Likewise, a meta-analysis revealed that the use of berberine combined with lifestyle interventions lowered fasting plasma glucose, postprandial glucose, and $\mathrm{HbAlc}$ as compared with lifestyle interventions alone. ${ }^{4}$ Using berberine with lifestyle interventions or combined with oral hypoglycemic medications also appears to be safe and effective in clinical studies as an adjunctive therapeutic. ${ }^{4}$

In addition, $\alpha$-lipoic acid (ALA), a powerful antioxidant, has been shown to reduce peripheral neuropathy in $\mathrm{T}^{2} \mathrm{DM} .{ }^{6,7} \mathrm{~A}$ review conducted in 2011 found oral or parenteral supplementation of $600 \mathrm{mg}$ ALA safely and effectively reduced signs and symptoms of diabetic sensorimotor polyneuropathy. ${ }^{6}$ Likewise, a study conducted in 2018 found a reduction of neurological symptoms in patients with diabetic neuropathy. ${ }^{7}$ The mechanism for improvements may be attributed to the restoration of thiol homeostasis (ALA is a source of thiol), counteraction of the oxidative process, and/or action as an insulin mimetic. ${ }^{6,7}$

Fish oils have been shown to protect against diabetic retinopathy as well as increase high-density lipoprotein cholesterol and decrease low-density lipoprotein (LDL) cholesterol, triglycerides, and diastolic blood pressure. ${ }^{8}$ In middle-aged and older individuals with T2DM, intake of at least $500 \mathrm{mg}$ per day of dietary long-chain $\omega-3$ polyunsaturated fatty acids is associated with a decreased risk of diabetic retinopathy. ${ }^{9}$

With an emphasis on diet, incorporating low glycemic index foods, regular exercise, and nutraceutical supplementation, a holistic treatment plan can be devised. This integrative approach can accommodate personal and cultural food preferences, activity level, and symptoms while maintaining appropriate caloric needs for diabetes management. ${ }^{1}$

\section{Case Description}

A 45-year-old Caucasian female presented with chief concerns of diabetes, obesity, neuropathy, polyuria, and polydipsia in June 2016. She was diagnosed with T2DM in 2012 at which time, metformin therapy was initiated, but was discontinued due to dizziness, abdominal pain, and nausea. The patient reported managing her blood glucose with lifestyle interventions, including exercise, diet, and supplementation.

The patient reported that her blood glucose readings had consistently been $>200 \mathrm{mg} / \mathrm{dL}$ in the morning and between 90 and $100 \mathrm{mg} / \mathrm{dL}$ in the evening. Her highest reading recorded was $320 \mathrm{mg} / \mathrm{dL}$ in the morning. She reported traveling globally for work several months of the year and as a result stated that she did not always have access to healthy food options. She reported constant tingling and discomfort in both feet that began six weeks ago before her initial visit. In addition, she reported urinary urgency and frequency, nocturia, polydipsia, and polyphagia. Cardiovascular and respiratory examinations were unremarkable. Skin examination revealed mild nonpitting edema of the pretibial region and generalized skin tags over the neck and shoulders. A sensory deficit in distinguishing between sharp and dull was determined on the left foot in all dermatomes. No ulceration of the feet was noted. Written informed consent was obtained from the patient for publication of this case report.

\section{Investigation}

Laboratories revealed an elevated fasting blood glucose (FBG) of $351 \mathrm{mg} / \mathrm{dL}, \mathrm{HbA} 1 \mathrm{c}$ of $12.8 \%$, cholesterol at $207 \mathrm{mg} /$ $\mathrm{dL}$, and $\mathrm{LDL}$ at $134 \mathrm{mg} / \mathrm{dL}$. Vitamin D was borderline low at $31.8 \mathrm{ng} / \mathrm{mL}$. All other laboratory findings, including complete blood count, complete metabolic panel, C-reactive protein, microalbumin, creatinine, thyroid-stimulating hormone, free T4, fasting insulin, GAD-65, and islet cell antibodies (ICA), were within normal limits.

\section{Treatment}

Given the patient's severity of the laboratory abnormalities for $\mathrm{HbAlc}$ and blood glucose, a conventional approach would likely have included prescription of insulin therapy. Insulin is considered a first-line therapy for all patients with T2DM, particularly for patients presenting with $\mathrm{HbA} 1 \mathrm{c}$ levels that are relatively far from the goal (e.g., $>9 \%$ ). ${ }^{10}$ Metformin is suggested as an initial therapy in most patients; however, sulfonylureas and sodium-glucose cotransporter-2 (SGLT2) inhibitors can be used for patients with intolerances to metformin.

Conventional and naturopathic models emphasize that patients with newly diagnosed diabetes should participate in a comprehensive diabetes self-management education program, which includes instruction on nutrition, physical activity, optimizing metabolic control, and preventing complications. ${ }^{10}$ For highly motivated patients with A1c near target (i.e., $<7.5 \%$ ), a three- to six-month trial of lifestyle modification before initiating pharmacologic therapy is reasonable. For most patients presenting with Alc at or above target level (i.e., $>7.5 \%-8 \%$ ), pharmacologic therapy should be initiated at the time of diabetes diagnosis.

We utilized a shared decision-making model to determine the appropriate personalized intervention for the patient (Table 1). We prescribed metformin in a titration of $250 \mathrm{mg}$ by mouth (po) one time per day (q.d.), progressing to $500 \mathrm{mg}$ po q.d. and then to $500 \mathrm{mg}$ po two times per day (b.i.d.) with each titration increase in seven-day intervals to attempt to reduce adverse gastrointestinal side effects. In addition, we prescribed berberine $500 \mathrm{mg}$ po b.i.d. and fish oils $2 \mathrm{~g}$ po q.d. to address the hypercholesterolemia. Vitamin $\mathrm{D}_{3}$ at 5000 IU po q.d. was advised to correct the underlying vitamin D insufficiency, and $100 \mathrm{mg}$ of ALA po b.i.d. was recommended to target her peripheral neuropathy. We encouraged the patient to follow a Mediterranean 


\section{Table 1. Supplement and Medication Modifications}

\begin{tabular}{|c|c|}
\hline $\begin{array}{l}\text { Oral supplement } \\
\text { and medication } \\
\text { regimen }\end{array}$ & Dosage \\
\hline Metformin & $\begin{array}{l}250 \mathrm{mg} \text { q.d. } \times 7 \text { days, then } \\
500 \mathrm{mg} \text { q.d., then } 500 \mathrm{mg} \text { b.i.d. }\end{array}$ \\
\hline Berberine & 500 mg b.i.d. (2 caps) \\
\hline$\omega-3$ Fatty acids & 2 g q.d. (2 caps) \\
\hline Vitamin $D_{3}$ liquid & 5000 IU q.d. (5 drops) \\
\hline$\alpha$-Lipoic acid & 100 mg b.i.d. (2 caps) \\
\hline
\end{tabular}

diet and avoid processed foods. We recommended small frequent meals with at least $15 \mathrm{~g}$ of protein per meal. Lastly, a daily exercise regimen involving 30-60 minutes of moderate-intensity aerobic activity was emphasized for weight management and improved glycemic control. Since the patient reported swimming, we provided an individualized recommendation to swim 15 laps in the pool three times per week over the next two weeks and increase to 20 laps three times per week thereafter.

\section{Outcomes and Follow-Up}

At three months follow-up, the patient's signs, symptoms, and laboratory results changed considerably. The patient reported adhering to the treatment plan as prescribed, although she had self-discontinued metformin one month after initiating therapy due to adverse gastrointestinal effects. Follow-up laboratory analysis demonstrated a reduction in $\mathrm{HbAlc}$ from $12.8 \%$ to $6.8 \%$. Likewise, FBG decreased from the initial reading of 351 to $157 \mathrm{mg} / \mathrm{dL}$ over the three-month intervention period. Her subjective energy increased, and she reported that she no longer experienced neuropathy in her feet.

Our patient returned after several months of traveling for work and reported a relapse in her diet, decrease in exercise, and inconsistency with her supplements. Several of her symptoms returned, including weight gain, polyuria, and polyphagia. Laboratory analysis during a four-month followup visit demonstrated elevated blood glucose of $298 \mathrm{mg} / \mathrm{dL}$, cholesterol of $204 \mathrm{mg} / \mathrm{dL}$, triglycerides of $204 \mathrm{mg} / \mathrm{dL}$, LDL of $122 \mathrm{mg} / \mathrm{dL}$, and $\mathrm{HbAlc}$ of $9.8 \%$. At this point, our initial dietary and lifestyle modifications were represcribed, as well as initiation of therapy with the SGLT2 inhibitor dapagliflozin $10 \mathrm{mg}$ tablet po q.d., which our patient reported was better tolerated than metformin.

\section{Discussion}

At three months follow-up, the patient's laboratories improved dramatically and she reported significant improvements in her signs and symptoms. After a reported relapse, her outcomes, although still not at goal, remained improved over initial measures. This case elucidates the possibility of successfully managing diabetes with lifestyle, nutraceutical, and pharmacologic interventions without the use of insulin and highlights the need for further research on holistic approaches to diabetes management. It also highlights the importance of compliance.

There are several plausible explanations as to why our patient responded so favorably to this integrative intervention. Her response to therapy could be attributed to a particular subset of diabetes, which may have increased her responsiveness to dietary and lifestyle changes. Although she was tested for latent autoimmune diabetes in adults through GAD-65 and ICA, the laboratories were negative for this condition. Other subsets of diabetes are not yet easily measured, so this theory could not be ruled out entirely. Future research may aim to identify which subtypes are likely to respond to lifestyle-based interventions.

In addition, the plausibility of synergistic effects of supplementation, physical activity, diet, and/or metformin may have positively affected outcomes in this study. Synergistic and/or combination therapeutic effects on diabetes and diabetic-related conditions have yet to be explored in large validating clinical trials. Further investigation is needed to determine the safety and efficacy of these therapeutic approaches.

Finally, adherence to lifestyle modifications is necessary to demonstrate long-term management of blood sugar levels. Many lifestyle interventions require significant health behavior changes, thus these therapies may not be effective for everyone. This issue should be considered in study design to identify target populations who are most likely to adhere to a holistic treatment plan.

We have demonstrated that it is possible to successfully treat patients with severely uncontrolled T2DM with diet, exercise, and medications without immediately prescribing insulin. We must be careful not to overgeneralize these results as questions remain regarding the effect of diabetes subtypes and synergy of therapies on health outcomes as well as the feasibility of strict adherence to such protocols. Future research may investigate these areas to advance our understanding of holistic treatment plans for diabetes management.

\section{Author Disclosure Statement}

No competing financial interests exist.

\section{Funding Information}

This research did not receive any specific grant from any funding agency in the public, commercial, or not-for-profit sector.

\section{References}

1. Ley SH, Hamdy O, Mohan V, Hu FB. Prevention and management of type 2 diabetes: Dietary components and nutritional strategies. Lancet 2014;383:1999-2007. 
2. Hemmingsen B, Gimenez-Perez G, Mauricio D, et al. Diet, physical activity or both for prevention or delay of type 2 diabetes mellitus and its associated complications in people at increased risk of developing type 2 diabetes mellitus. Cochrane Database Syst Rev 2017;12:CD003054.

3. Chen L, Jian-Hao P, Jian K, et al. Effect of lifestyle intervention in patients with type 2 diabetes: A meta-analysis. Metabolism 2015;64:338-347.

4. Lan J, Zhao Y, Dong F, et al. Meta-analysis of the effect and safety of berberine in the treatment of type 2 diabetes mellitus, hyperlipemia and hypertension. J Ethnopharmacol 2015;23:69-81.

5. Mahmoud MA, Ghareeb DA, Sahyoun HA, et al. In vivo interrelationship between insulin resistance and interferon gamma production: Protective and therapeutic effect of berberine. Evid Based Complement Alternat Med 2016;2016:1-7.

6. McIlduff CE, Rutkove SB. Critical appraisal of the use of alpha lipoic acid (thioctic acid) in the treatment of symptomatic diabetic polyneuropathy. Ther Clin Risk Manag 2011;7:377-385.

7. Vural G, Bektas H, Gumusyayla S, et al. Impaired thiol-disulphide homeostasis in patients with axonal polyneuropathy. Neurol Res 2018;40:166-172.

8. Shen T, Xing G, Zhu J, et al. Effects of 12-week supplementation of marine omega-3 PUFA-based formulation omega3Q10 in older adults with prehypertension and/or elevated blood cholesterol. Lipids Health Dis 2017;16:253.
9. Chew EY. Dietary intake of omega-3 fatty acids from fish and risk of diabetic retinopathy. JAMA 2017;317:2226

10. McCulloch D. Initial management of blood glucose in adults with type 2 diabetes mellitus. UpToDate 2017. Online document at: https://www.upto date.com/contents/initial-management-of-blood-glucose-in-adults-with-type-2diabetes-mellitus Accessed November 22, 2019.

Kendall Smith, ND, is a naturopathic doctor and first year resident at Bastyr University Clinic in San Diego, California. Jacob Hwang, ND, is a naturopathic doctor at Bastyr University Clinic and adjunct faculty member in the School of Naturopathic Medicine at Bastyr University California in San Diego, California. Shawna McNally, MSPH, RD, is a published public health consultant at Accents on Health in San Diego, California. Ryan McNally, ND, MS, PA, is a naturopathic doctor and physician assistant. He is the chief medical officer at Bastyr University Clinic and core faculty member in the School of Naturopathic Medicine at Bastyr University California in San Diego, California.

To order reprints of this article, contact the publisher at (914) 740-2100. 